\title{
Legorization from silhouette-fitted voxelization
}

\author{
Kyungha Min ${ }^{1}$, Cheolseong Park ${ }^{1}$, Heekyung Yang ${ }^{1}$ and Grim Yun ${ }^{2}$ \\ ${ }^{1}$ Dept. of Computer Science, Graduate School, Sangmyung Univ., \\ Hongji-dong, Jongro-gu, Seoul, Korea \\ [e-mail: minkh@smu.ac.kr, tjd4987|yhk775206@naver.com] \\ ${ }^{2}$ Dept. of Computer Science, Sangmyung Univ., \\ Hongji-dong, Jongro-gu, Seoul, Korea \\ [e-mail: rmfla0214@ naver.com] \\ *Corresponding author: Grim Yun
}

Received September 21, 2017; revised December 15, 2017; revised February 9, 2018; accepted February 18, 2018; published June 30, 2018

\begin{abstract}
We present a legorization framework that produces a LEGO model from user-specified 3D mesh model. Our framework is composed of two stages: voxelization and legorization. In the voxelization, input 3D mesh is converted to a voxel model. To preserve the shape of the 3D mesh, we devise a silhouette fitting process for the initial voxel model. For legorization, we propose three objectives: stability, aesthetics and efficiency. These objectives are expressed in a tiling equation, which builds a LEGO model using layer-by-layer approach. We legorize five models including characters and buildings to prove the excellence of our framework.
\end{abstract}

Keywords: legorization, LEGO, voxelization

The early version of this paper was presented at Computer Graphics International 2017 as a short paper. 


\section{Introduction}

LEGO is a well-known assembly toy from the LEGO company in Denmark. The LEGO consists of colorful interlocking plastic bricks with many other parts such as gears and minifeatures. The most commonly used bricks are of rectangular shape with various lengths, widths and heights.

Sometimes, many LEGO users have a desire to assemble their own models, whose assembly diagram is not provided by the company. Only highly experienced LEGO users design their own diagram to build a LEGO model for their desired models. Recent progress of digital contents such as games or animations accelerates the desire to build a LEGO model for their favorite characters, e.g. Eevee of Pokémon.

We present a framework to build an assembly diagram for a user-selected 3D mesh model that guides users to build their LEGO model using commercial LEGO bricks. Our framework is composed of two stages: voxelization and legorization.

Many existing legorization works start from a voxel model which is converted from a 3D mesh. Like these works, we employ an existing slice-based voxelization scheme to produce an initial voxel model. Furthermore, we present a silhouette-based fitting scheme for the initial voxel model to achieve a voxel model whose shape is as similar as possible to the input 3D mesh.

Legorization is defined as a process that tiles the voxel model using a series of bricks. We employ a layer-based approach that fills the voxel model layer by layer using appropriate LEGO bricks. Unlike other frameworks, we include bricks whose height is more than one layer. We have three objectives for legorization: stability, aesthetics and efficiency. We define a tiling equation that covers these objectives and find the solution of the equation using a heuristic search algorithm. Our framework is distinguished in these points:

1. Shape-preserving voxelization: To preserve the shape of an input 3D mesh, we design a voxelization process that modifies the initial voxel model by fitting the silhouette of an initial voxel model to that of the input 3D mesh.

2. Objective-driven legorization: We pursue three objectives including stability for a stable structure, aesthetics for a balanced layout and efficiency for using multi-height bricks. Multi-height bricks have not been considered in the existing works.

3. Efficient data structure and implementation: We devise a two-colored graph that represents the adjacency and stability information of a LEGO model. Our legorization is composed of tiling process on each layer, which is implemented using a heuristic search algorithm.

We illustrate the overview of our algorithm in Fig. 1. An input 3D model is converted to an initial voxel model, which is further adjusted to the input model using our silhouette fitting scheme. Each layer of the voxel model is tiled through the LEGO bricks using an energy minimization scheme that optimizes users' aimed targets such as stability, aesthetics and efficiency. 


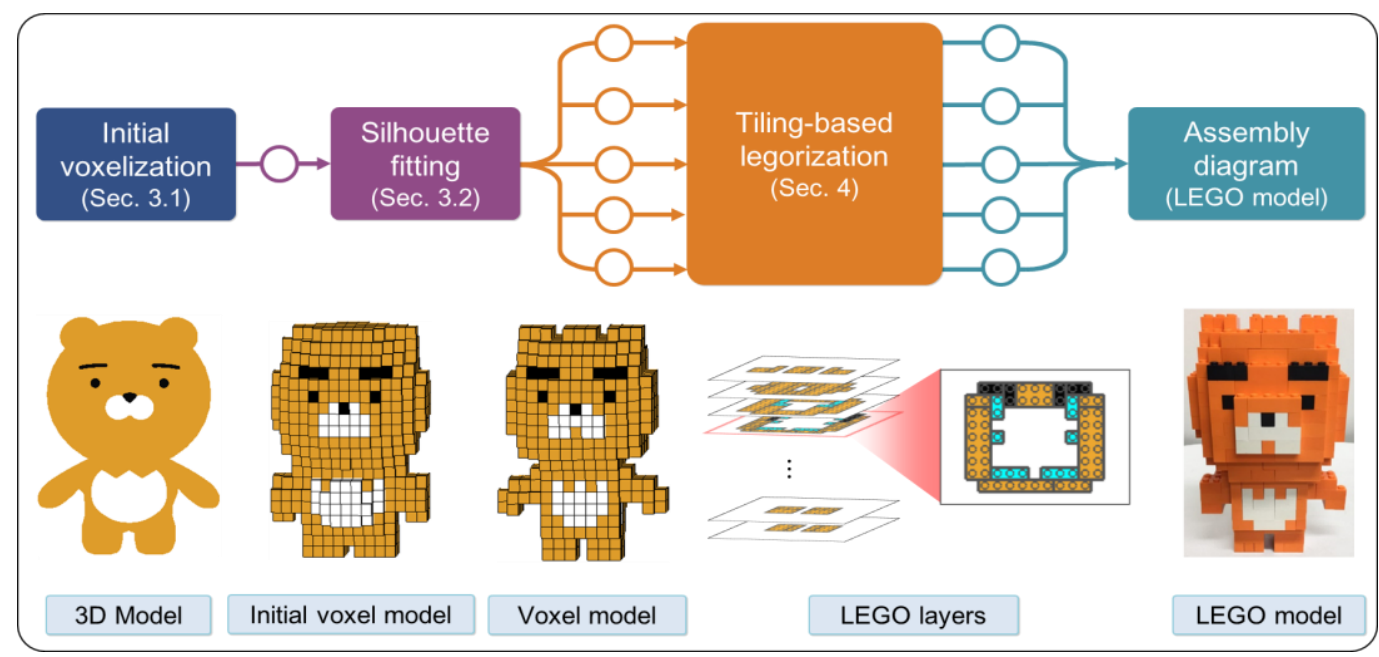

Fig. 1. Overview of our algorithm.

\section{Related Work}

\subsection{Voxelization techniques}

Voxelization techniques are originated from 1980s. Kaufman et al. presented a voxelization algorithm that converts continuous geometric objects into an array of voxels in 3D discrete space while preserving their shapes $[1,2]$.

The progress of GPU motivates an acceleration scheme for voxelization algorithms using the GPUs. Fang and Chen $[3,4]$ presented a scheme that reconstructs volume model from the sliced image, which is created by intersecting an input 3D model with a clipping plane using GPU computation. Karabassi et al. [5] also presented a voxelization scheme employing GPU and a depth-buffer supported by OpenGL library. Passalis et al. [6] added a stencil buffer to the scheme in [5] to present a lossless voxelization scheme for an arbitrary surface model. Eisemann and Decoret [7] presented an accelerated scene voxelization scheme using GPU. They defined an ambient lattice on a rendered scene from a viewpoint. Furthermore, the depth information is stored in the RGBA channel to reduce the computation time for shade rendering and reflection rendering. They [8] also presented a single pass approach for voxelizing the inside of a watertight model, which guarantees real-time voxelization for high-resolutional models.

There are also several voxelization schemes that do not rely on GPU. Silva et al. [9] presented a scheme that renders a mesh model as a voxelized model using a point sampling scheme that segments the edges of a triangular mesh according to the user-initiated resolution. Nooruddin and Turk [10] employ a ray casting to count the number of intersection points on a target model and a parity counting to build a voxelized model. Finally, they modify the voxel model using a series of morphological operators.

Recently, real-time voxelization algorithms using GPU acceleration are presented for high-resolutional models. Hong et al. [11] reduce the inefficient computation on the empty voxels lying inside of a surface model by constructing an octree structure. Schwarz and Seidel [12] presented a sparse voxel octree(SVO) structure to voxelize a solid model. 


\subsection{Legorization techniques}

\subsubsection{Early techniques}

Gower et al. [13] pioneered a computerized framework for generating a LEGO model from a polygonal model. They define several heuristics for designing LEGO model and propose a minimization scheme for a penalty function using simulated annealing. The heuristics they define include cover ratio, big brick, perpendicularity, vertical boundary, T-shaped joining, and covered edge by center (See Appendix for an explanation). Their framework, however, has not been implemented in their paper. Petrovic [14] employed a genetic algorithm to resolve the LEGO construction problem and van Zijl and Smal [15] used cell clustering strategy to merge $1 \times 1$ bricks to design a LEGO layout. Later, Smal [16] improved this scheme by applying a beam search algorithm. These works use primitive data structure such as a list ([14]) and a grid ([15, 16]).

\subsubsection{Recent techniques without physical stability}

Testuz al. [17] presented a graph-based randomized greedy algorithm to merge $1 \times 1$ bricks to general LEGO bricks of diverse scales. A LEGO model is organized as a graph whose vertex corresponds to the brick and edge to the connectivity. Therefore, an articulation point of the graph represents a brick where many parts of the model are connected. Their strategy is to build a biconnected graph where an articulation point does not exist. They do not go into physical problem considering the weights of the bricks and gravity. Ono et al. [18] presented a graph-based greedy merging algorithm for build a LEGO design. Their strategy resembles Testuz et al.'s work [17]. Their merging algorithm includes the heuristics defined by Gower et al. [13] such as big brick and cover ratio. Their algorithm, however, has several degenerate cases for connecting the bricks. Zhang et al. [19] presented a construction scheme for planar LEGO model using pattern tiling. An input image is pixelized and the neighboring pixels of similar colors are merged to form various LEGO bricks. Kim et al. [20] surveyed the existing LEGO representation schemes and their construction algorithms. Lee et al. [21] combined a greedy algorithm and a genetic algorithm to improve the layout of LEGO bricks. Zhang et al. [22] proposed a random merge scheme considering stability, symmetry and color separation. This scheme may take a lot of computation time due to their randomized merging strategy. Stephenson [23] proposed a four-phase search algorithm to build a LEGO model. The phases include arrangement according to the direction, edge reduction, brick recombination and building single component. Since each merging step takes four-phase search, this scheme suffers from a long computation time. Zhang et al. [24] improved their previous layer-by-layered assembly approach, which is not appropriate for over-hanging blocks. Instead of legorizing a polygonal model, they proposed an alternative approach that assembles the pre-built components. Hong et al. [25] improved Testuz et al.'s greedy approach [17] by adjusting the centroid of the bricks

These works commonly employed a merging approach that merges $1 \times 1$-sized bricks into bricks of various sizes. These merging approaches, however, leave $1 \times 1$ bricks very frequently and the final layout fails to present balanced and aesthetic visualization. Therefore, we employ a tiling approach that tiles various sized bricks at their appropriate locations, which prevents small-sized bricks and global visualization considerations. 


\subsubsection{Recent techniques with physical stability}

Luo et al. [26] presented a LEGO model generation framework that considers physical stability using force-based analysis, where the vertical and horizontal friction force of a brick are considered with maximum friction load of the brick. They aim to build a singly connected component of the graph representation of the LEGO model. In the merging process, the force loaded on each brick is computed and the stability is checked. This approach sometimes cannot find a physically stable LEGO model from input 3D mesh models. Kozaki et al. [27] presented a LEGO model generation scheme using photographs taken from a physical object in various angles. They also proposed an energy minimization scheme for LEGO model generation. Their energy function includes momentum, which provides physical stability of the model. To build a light-weighted model for portability, they devised a hollowing process to empty the inside of the model.

We do not consider the physical computation in our approach, since most of the 3D models such as characters and buildings we test are balanced. Some unbalanced characters are the target of our future work.

\section{Voxelization}

\subsection{Initial voxelization}

We start our voxelization process from a user-specified 3D mesh by rendering the mesh using the rendering pipeline of OpenGL library [4]. By controlling a clipping plane of the rendering pipeline, we extract a series of slice images, which are mapped into a layer information of our voxel model. This layer information is stacked to form our initial voxel model.

Since the resolution of the voxelization is limited, the shape of the initial voxel model can be far from the shape of the 3D mesh. To fit the initial voxel model as accurate as possible, we propose a silhouette fitting process, which carves the silhouette of the initial voxel model to fit that of the input 3D mesh.

We extract the silhouettes of the initial voxel model and the input 3D mesh by rendering them using a color distinguishable from their background. The rendered image stored in a framebuffer is bi-colored, which distinguishes the inside and outside of a silhouette. We visit the boundary of the model using a chain code algorithm to build a silhouette curve [28].

We build a signed distance map on the silhouette voxels from the silhouette curve. The distance is estimated as the closest distance between the center of the silhouette voxel and the closest point on the curve. We illustrate the distance estimation process in Fig. 2. Furthermore, we accelerate the distance estimation using jump flooding algorithm [29]. The distance value $d(v)$ stored at a voxel $\boldsymbol{v}$ is estimated as follows:

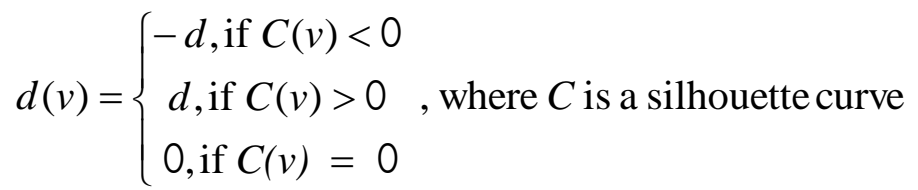




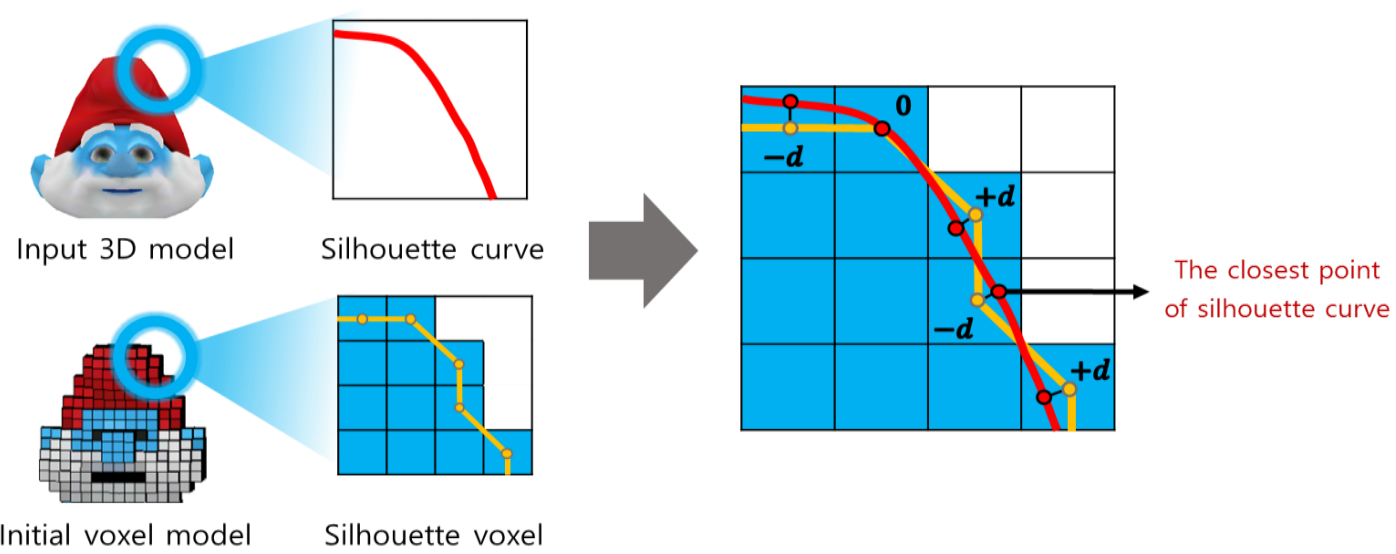

Fig. 2. The distance estimation process: At each silhouette voxel, the signed distance to the silhouette curve is estimated.

\subsection{Silhouette fitting}

\subsubsection{Approximation of differentiation}

To fit the initial voxel model to the input 3D mesh, we match the silhouette voxels of the initial voxel model to its closest silhouette pixel of the 3D mesh. After matching, we estimate the first-order differentiations $\left(\boldsymbol{t}\left(v_{i}\right)\right)$ and the second-order differentiations $\left(\boldsymbol{g}\left(v_{i}\right)\right)$ at the matching voxels. We use a central difference approximation for the differentiation. Since the $v$ is an approximation estimated on a silhouette curve, unwanted anomaly may appear. To remedy this, we sample three candidates for the differentiation: one-ring neighborhood difference $\left(v_{i-1}\right.$ and $\left.v_{i+1}\right)$, two-ring neighborhood difference $\left(v_{i-2}\right.$ and $\left.v_{i+2}\right)$ and three-ring neighborhood difference $\left(v_{i-3}\right.$ and $\left.v_{i+3}\right) . \boldsymbol{t}\left(v_{i}\right)$ and $\boldsymbol{g}\left(v_{i}\right)$ are determined by averaging three candidate values.

\subsubsection{Energy function}

The energy function that defines the shape difference between the silhouettes of the voxel model and the input 3D mesh is defined as follows:

$$
E(V, S)=\sum_{v \in V}\left(d(v, S)+\left(1-t(v) \cdot t\left(S_{v}\right)\right)+\left(1-g(v) \cdot g\left(S_{v}\right)\right)\right),
$$

where $V$ is the voxel model, $S$ is the 3D mesh model, and $S_{v}$ is a pixel on a chain code from the 3D mesh silhouette, which is closest to $v \in V$. is an inner product operator. The distance of $v$ from the 3D mesh silhouette, denoted as $d\left(v, S_{v}\right)$, is defined as follows:

$$
d\left(v, S_{v}\right)=(d(v)>0) ? d(v): 0
$$

The first term of Eqn (2), $d(v, S)$, becomes zero, if the voxel is not outside of the silhouette. This term may lead to a shrunk voxel model. To avoid this, we add the second term, $1-\boldsymbol{t}(v)$. $\boldsymbol{t}\left(S_{v}\right)$, and the third term, $1-\boldsymbol{g}(v) \cdot \boldsymbol{g}\left(S_{v}\right)$. These terms estimate the difference of the tangents and gradients of the models, respectively. The second term becomes zero, if the tangents from both models are identical, and the third term becomes zero, if the gradients are identical. We illustrate this in Fig. 3. 


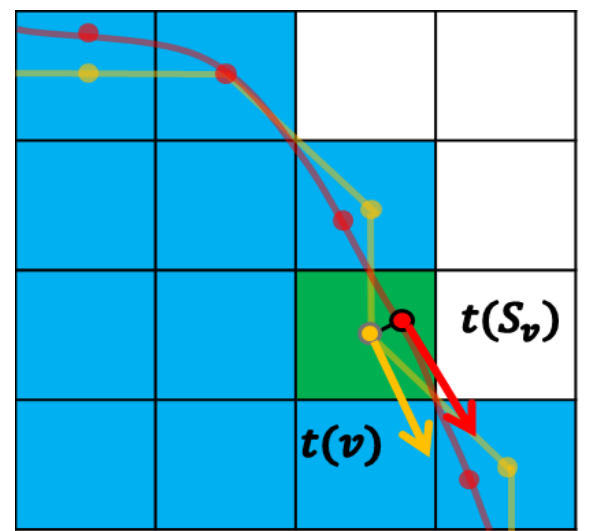

Fig. 3. The tangent vectors of the energy function

\subsubsection{Minimization}

We fit the initial voxel model to the 3D mesh model by carving the voxels in the silhouette of the initial voxel model by minimizing $E(V, S)$. For an efficient minimization, we sort the voxels $v$ 's on the silhouette according to the descending order of $d(v)$ and store them in a priority queue. We select the voxel $w$ in the top of the priority queue, and compare $E(V-\{w\}$, $S)$ and $E(V, S)$. If $E(V-\{w\}, S)<E(V, S)$, then we remove $w$ from $V$ and the priority queue and update $V$ by inserting new silhouette voxels. Otherwise, we remove $w$ only from the priority queue. We repeat this process until the priority queue is empty. We illustrate two cases in Fig. 4.
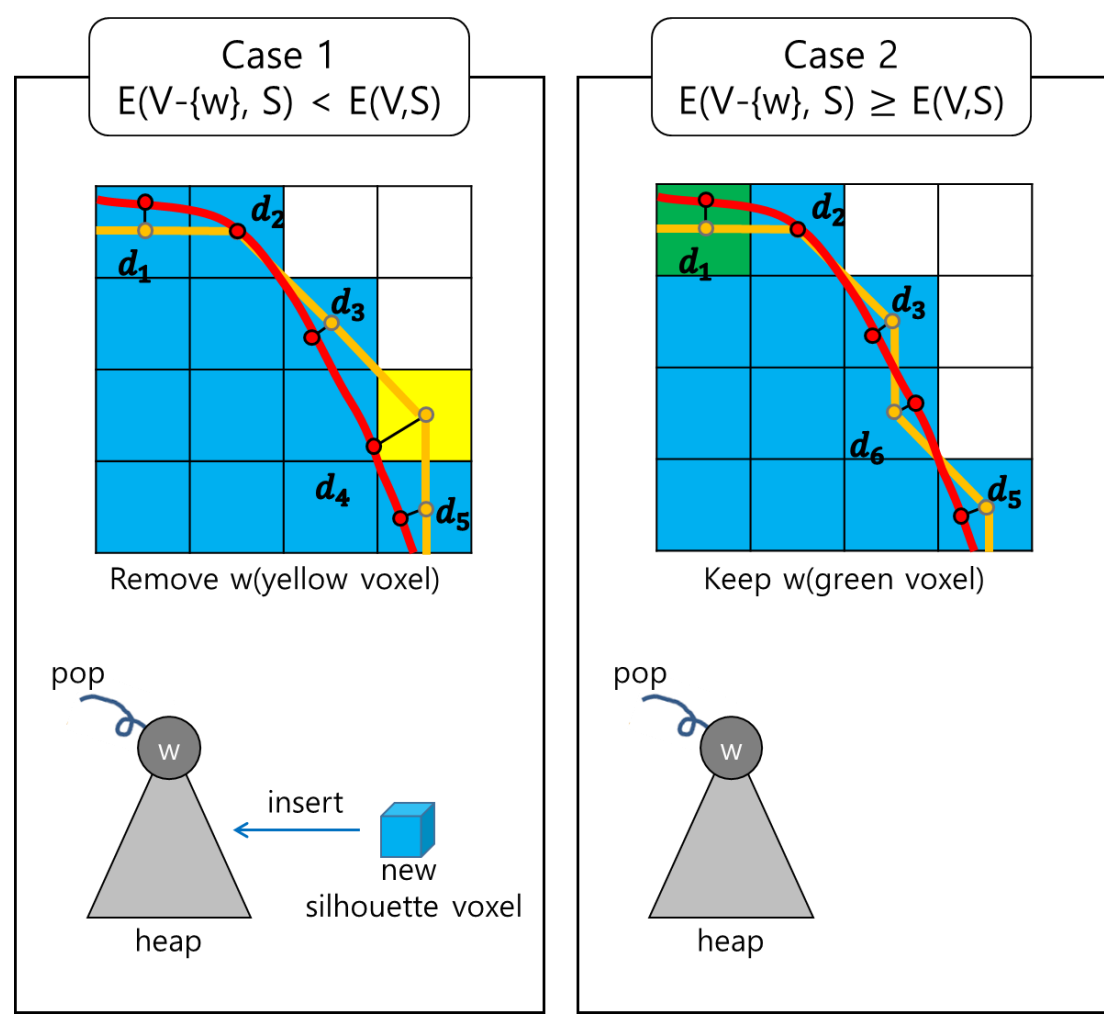

Fig. 4. Two cases of energy minization: In Case 1, the target voxel (rendered in yellow) is removed from the voxel model and a new silhouette voxel is added instead. In Case 2, the target voxel (rendered in green) is preserved. 
The pseudo code for the energy minimization is presented below:

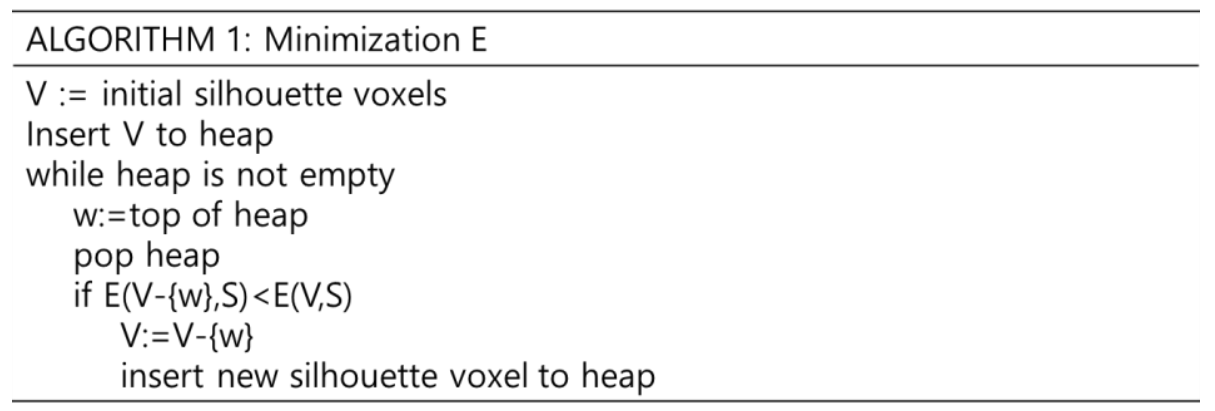

We present our voxelization results in Fig. 5.

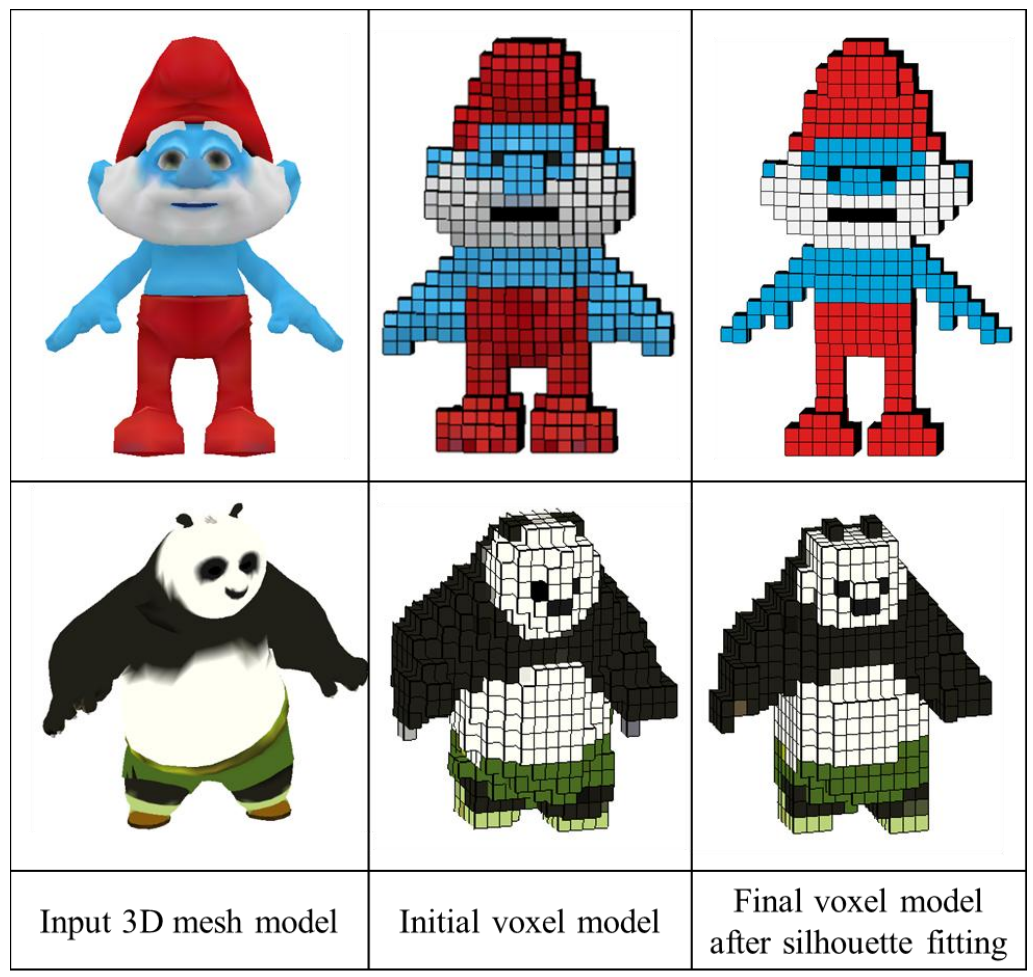

Fig. 5. Voxelization results.

\section{Legorization}

\subsection{Model representation}

\subsubsection{Model definition}

Since Testuz et al. [17] employed a graph structure to represent a LEGO model, some researchers followed this approach $[27,21,26]$. In these approaches, a brick is mapped to a vertex and the combined bricks are to an edge. Therefore, two bricks in the same layer are not connected by an edge. By employing a graph structure, evaluating physical feasibility and 
stability of a LEGO model becomes the well-studied graph problems such as checking connected component or finding an articulation point. A graph for a LEGO model separated by several parts is disconnected, and a brick where too heavy weight is loaded is an articulation point. Therefore, they merge $1 \times 1$ bricks repeatedly to avoid these problems. Since their approaches use a merging strategy, the adjacent bricks in a layer can be identified by a simple index-based scheme. Since we employ a tiling approach, we cannot employ the graph structure used by the existing research

We propose a two-colored graph to represent our graph model. The key principle of our approach is to represent the adjacent bricks in the same layer as well as the combined bricks in the incident layer. Our two-colored graph is defined as follows:

1. Vertex: A brick is represented as a vertex.

2. Red edge: If two bricks are combined together in a LEGO model, then their corresponding vertices are connected by a red edge. A multi-height brick is represented as bricks of single height connected by a red edge.

3. Blue edge: If two bricks in a layer are adjacent, then their corresponding vertices are connected by a blue edge

Fig. 6 illustrates and compares our two-colored graph with the conventional graph proposed by Testuz et al. [17].

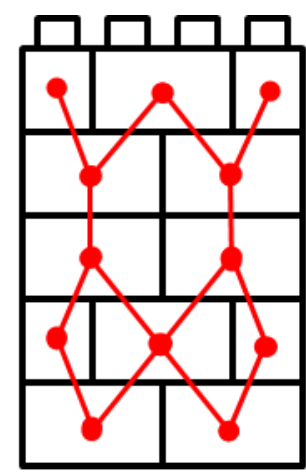

(a) Testuz et al. [24]

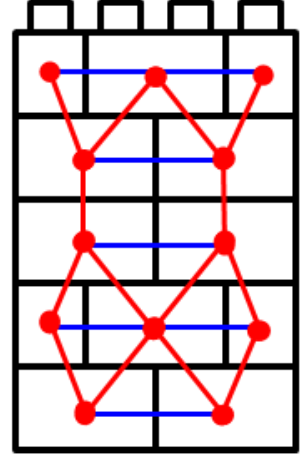

(b) Ours

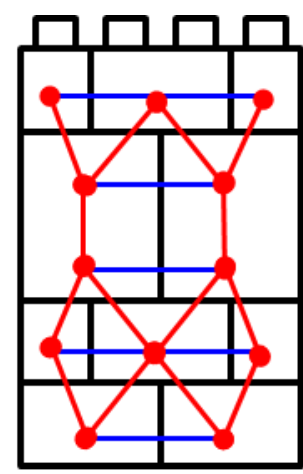

(c) Ours

(with multi-height bricks)

Fig. 6. Our two-colored graph and Testuz et al. [17]'s graph.

\subsubsection{Model construction}

Our legorization process is executed by constructing our two-colored graph for a voxel model. We design a layer-by-layer approach where our graph is constructed from the bottom layer of the voxel model. For each layer, we execute a tiling process that fills the layer with appropriate bricks. For each brick that covers the brick in the lower layer, a red edge that connects the two bricks is added to the graph. For the adjacent bricks, a blue edge that connects the two adjacent bricks is added to the graph. The tiling process is described in Section 4.2.

\subsection{Tiling}

We have three objectives in designing our tiling process for legorization: stability, aesthetics and efficiency. 
1. Stability is the most fundamental objective, which has been pursued from Gower et al. [13]. It means the constructed LEGO model is physically feasible and all the bricks in the model are combined

2. Aesthetics is the objective we pursue distinctively. A layer tiled by a LEGO artist is distinguished from automatically tiled layer by its balance, symmetry and accuracy. The bricks arranged aesthetically present greater interest than the bricks arbitrarily tiled

3. Efficiency is the time and cost requested to complete the target LEGO model. We pursue users to save their time and budget

\subsubsection{Stability}

We pursue the stability during the construction process of our two-colored graph using layer-by-layer approach. Gower et al. [13] proposed an edge cover heuristic to cover the edges in the lower layer with a brick to combine them tight and Testuz et al. [17] and other researchers $[21,26,18]$ proposed a connected component test and an articulation point search to check whether their LEGO model is stable or not. These schemes, however, restrict the freedom of choosing various tilings.

We propose a red-edge distance estimation on the two-colored graph for the stability. We estimate the distance of the adjacent bricks which are connected by a blue-edge only along red-edges (See Fig. 7). The red-edge distance, which is denoted as $\gamma$, reveals the minimum distance between the adjacent bricks and their combined point in a lower layer. According to the red-edge distance, we determine the priority for covering the adjacent bricks as follows:

1. Red-edge distance $(\gamma) \leq \boldsymbol{T}$ : The combined point of the adjacent bricks is not far, which means that the priority for covering is low.

2. Red-edge distance $(\gamma)>\boldsymbol{T}$ : The combined point of the adjacent brick is far, which means that the priority for covering is high.

3. Red-edge distance $(\gamma)=\infty$ : The adjacent bricks are not connected, which means that the priority for covering is highest.

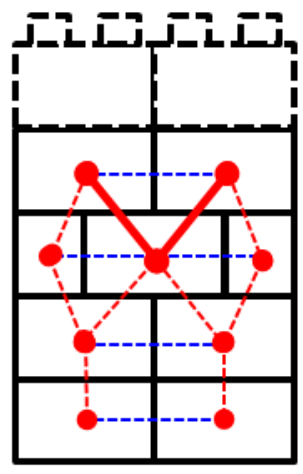

(a) $\gamma: 2, g_{s}=0.1$

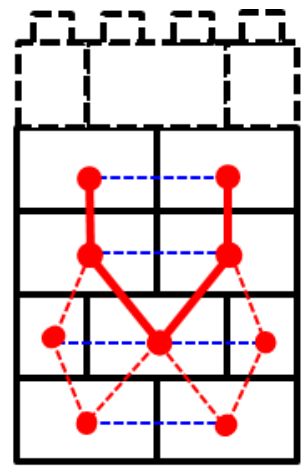

(b) $\gamma: 4, g_{s}=0.5$

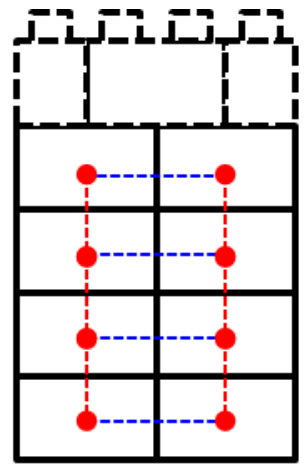

(c) $\gamma: \infty, g_{s}=1$

Fig. 7. Three cases of red edge distance $(\gamma)$ and their $g_{s}$ values.

Therefore, the stability term in our tiling equation becomes: 


$$
g_{s}=\frac{1}{m} \sum_{i=1}^{m} p(i), \text { where } p(i)=\left\{\begin{array}{c}
c(i) \times p_{1}, \text { if } \gamma \leq T \\
c(i) \times p_{2}, \text { if } \gamma>T \\
c(i) \times p_{3}, \text { if } \gamma \geq \infty \\
c(i), \text { otherwise }
\end{array}\right.
$$

,where $m$ denotes the number of blue-edges in the previous layer. $c(i)$ is 1 , if the $i$-th blue edge is not covered, and 0 , otherwise. In our implementation, we set $T$ as the height of our multi-height brick. $p_{1}$ is set as $0.1, p_{2}$ as $0.5, p_{3}$ as $1.0, T$ as 2 in this paper. Each priority is stored at blue-edges in our two-colored graph. Fig. 7 illustrates three cases of red edge distances and their $g_{s}$ values.

We compare the LEGO models produced by Gower et al. [13], by Testuz et al. [17] and ours in Fig. 8. The model presented in Fig. 8 (a) satisfies the heuristics proposed by Gower et al. [13] and (b) satisfies the heuristics from Testuz et al. [17]. From edge cover in [13], each edge is covered by the bricks in the above layer. Also the LEGO graph from [17] is a biconnected graph. Fig. 8 (c) presents our result. In tiling 4th layer, we estimate the red-edge distance and determine that the edges in the 3rd layer do not have a high priority to be covered. In tiling 5th layer, the red-edge distance increases, and the edges in the 4th layer have a higher priority to be covered. Therefore, we tile bricks in 5th layer to cover the edges in 4th layer. We can replace the consecutive bricks in 3rd and 4th layers to a multi-height brick.

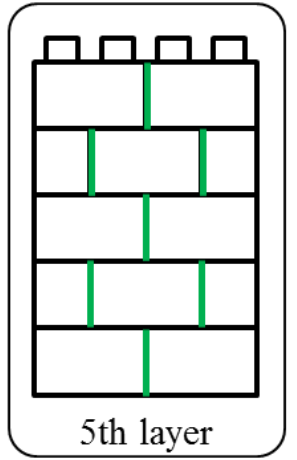

(a) Gower's [6]

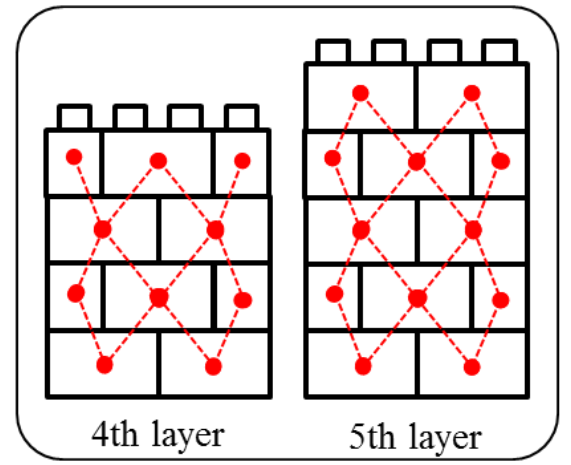

(b) Testuz et al.'s [24]

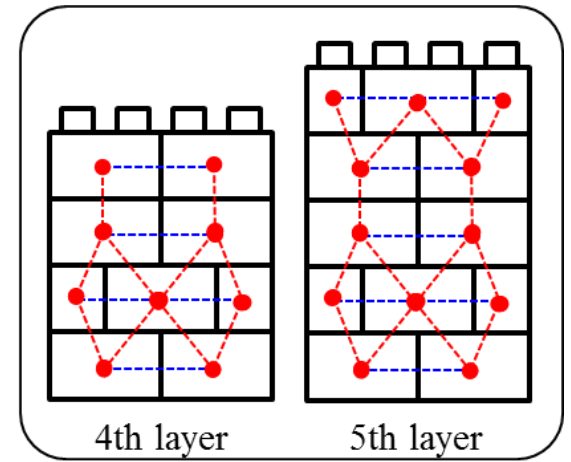

(c) Ours

Fig. 8. The comparison of tiling strategies for stability.

\subsubsection{Aesthetics}

Tiling LEGO bricks in a layer in a well-organized layout is a rarely pursued objective by the existing works. Zhang et al. [22] considered a symmetric layout of bricks, which constrains the bricks to be arranged along the axis of the model.

We propose a balanced tiling for aesthetics. As a preprocessing, we extract a central axis of the layer. Since our model is aligned with standard xyz-axis, we assume that each layer is aligned with the standard xyz-axis. Since z-axis is orthogonal to the layer, our axis is either $\mathrm{x}$-axis or $\mathrm{y}$-axis or both. Therefore, we estimate the balance for the three cases. A brick is balanced, if the axis passes through the center of the brick or there is a partner brick in the reflected coordinate for the axis. The term for the aesthetics is defined as follows: 


$$
g_{a}=\frac{1}{n} \sum_{i=1}^{n} b_{i}, \text { where } b_{i}=\left\{\begin{array}{c}
0, \text { if } i-\text { th brick is balanced } \\
1, \text { otherwise }
\end{array}\right.
$$

Fig. 9 illustrates the tiling that balances bricks according to the axis and estimates $g_{a}$.

\subsubsection{Efficiency}

Our LEGO model for a desired 3D model in the minimum cost and time is our third objective. We pursue this objective by saving the number of bricks in building the LEGO model. Many conventional researchers proposed big brick for their tiling principle that replaces several small bricks with a big brick as long as the tiling is stable. Our big brick is either horizontal or vertical.

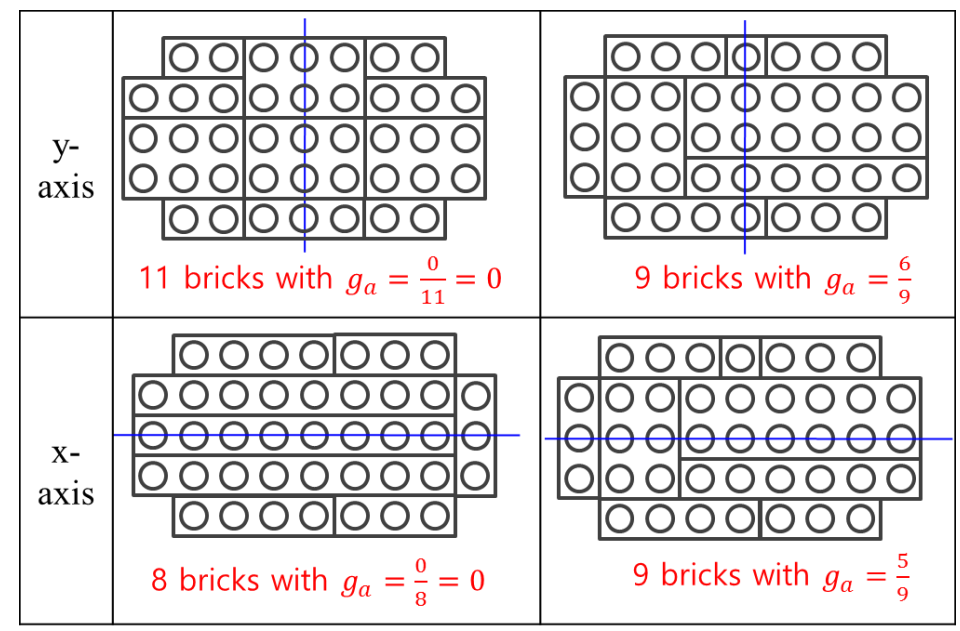

(a) Balanced tiling

(b) Unbalanced tiling

Fig. 9. The comparison of balanced tiling.

For horizontal big brick, we estimate the area of the brick and build our term as follows:

$$
g_{h}=\frac{1}{n} \sum_{i=1}^{n} \frac{A_{M A X}-A_{i}}{A_{M A X}-A_{M I N}},
$$

where $A_{M A X}$ is the area of maximum-sized brick and $A_{M I N}$ is the area of minimum-sized brick. In our paper, $A_{M A X}$ is 16 for a $8 \times 2$ brick and $A_{M I N}$ is 1 for a $1 \times 1$ brick. $A_{i}$ is the area of the $i$-th brick and $n$ is the number of bricks in a layer.

For a vertical big brick, we search two bricks that satisfy (i) same horizontal size, (ii) lying in the incident layers, and (iii) overlapped. These two bricks are merged into a multi-height brick. Therefore, the term for vertical big brick is defined as follows:

$$
g_{v}=\frac{1}{k} \sum_{i=1}^{k} v(i),
$$

where $v(i)$ is 0 , if the $i$-th brick is covered with a brick, which can be merged into a multi-height brick, and 1 , otherwise. Note that before estimating this term, we set $k$ as the number of the bricks in a layer that can be merged into a multi-height brick. Fig. 10 illustrates both horizontal and vertical big bricks for efficiency. 


\subsubsection{Tiling equation}

Our tiling equation for a $k$-th layer is the weighted sum of the terms defined in Eqn. (4) (7) we have defined:

$$
G\left(L_{k}\right)=w_{s} g_{s}+w_{a} g_{a}+w_{h} g_{h}+w_{v} g_{v},
$$

where $L_{k}$ is the set of bricks covering $k$-th layer.

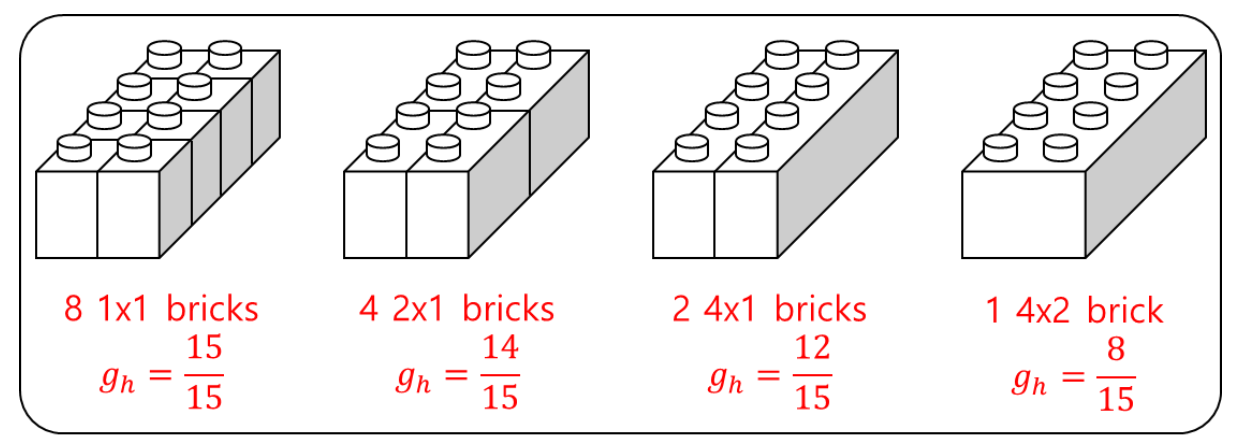

(a) Horizontal big brick

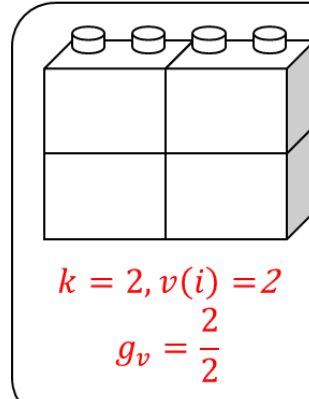

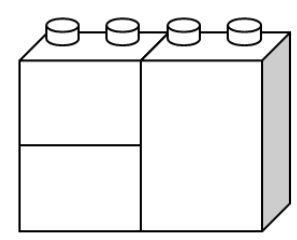

$k=2, v(i)=1$

$g_{v}=\frac{1}{2}$

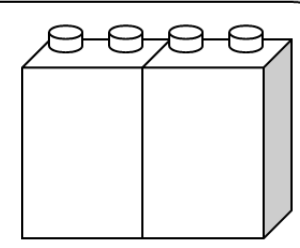

$k=2, v(i)=0$

$g_{v}=\frac{0}{2}$

(b) Vertical big brick

Fig. 10. Big bricks for efficiency.

Our layer tiling is executed from the bottom layer (1st layer) of the voxel model. In tiling $k$-th layer, we consider the $(k-1)$-th layer for the stability objective. The tiling equation in Eqn. (8) is minimized through $\mathrm{A}^{*}$ algorithm, one of the most widely used heuristic search algorithm. $\mathrm{A}^{*}$ algorithm starts at an empty layout where no brick is located. By considering the terms in the equation, the layer is filled with bricks. At every state of $\mathrm{A}^{*}$ algorithm, an $O P E N$ list and a CLOSED list are maintained and updated. OPEN list stores candidate configurations for minimization and CLOSED list stores configurations that cannot be minimized. Therefore, we choose one configuration from $O P E N$ list and continue tiling process.

In Fig. 11, we illustrate the process of tiling a layer by adding bricks one by one using the $\mathrm{A}^{*}$ algorithm. This process is implemented as building an A* search tree, where each node corresponds to a tiling status. During the search process, the value of the tiling equation for a node increases. This process terminates when a leaf node whose value is minimum is reached. By assigning different weights for the tiling equation in Eqn. (8), we achieve different layouts for the same layer. We discuss this problem further in Sec. 6.

Our problem of the $\mathrm{A}^{*}$ algorithm is that it may converge to a local minimum instead of global minimum. This problem can be avoided by searching the OPEN list in deeper levels. 
However, this strategy requires heavy computational time for tiling. Fortunately, the tiling executed in our samples did not show a problem of converting to a local minimum.

Another problem of the $\mathrm{A}^{*}$ algorithm is that the layout of global minimum may not be unique. Therefore, different layouts can be reached using the $\mathrm{A}^{*}$ algorithm. In this case, we choose one of the layout as our solution (See Fig. 12).

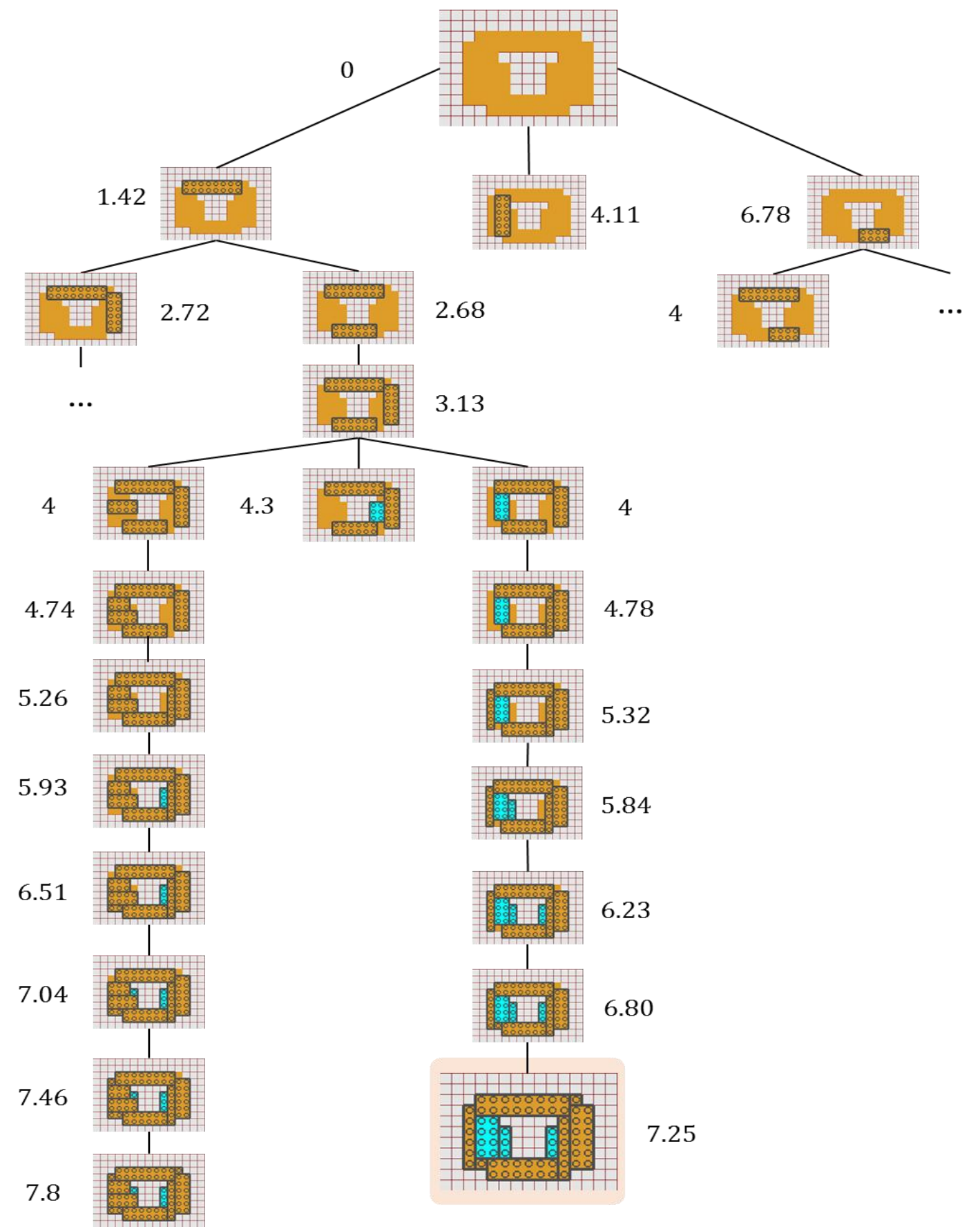

Fig. 11. An example of $A^{*}$ search tree. We reach a leaf node whose value from Eqn. (8) is 7.25 as minimum. The sample is from Ryan model. 


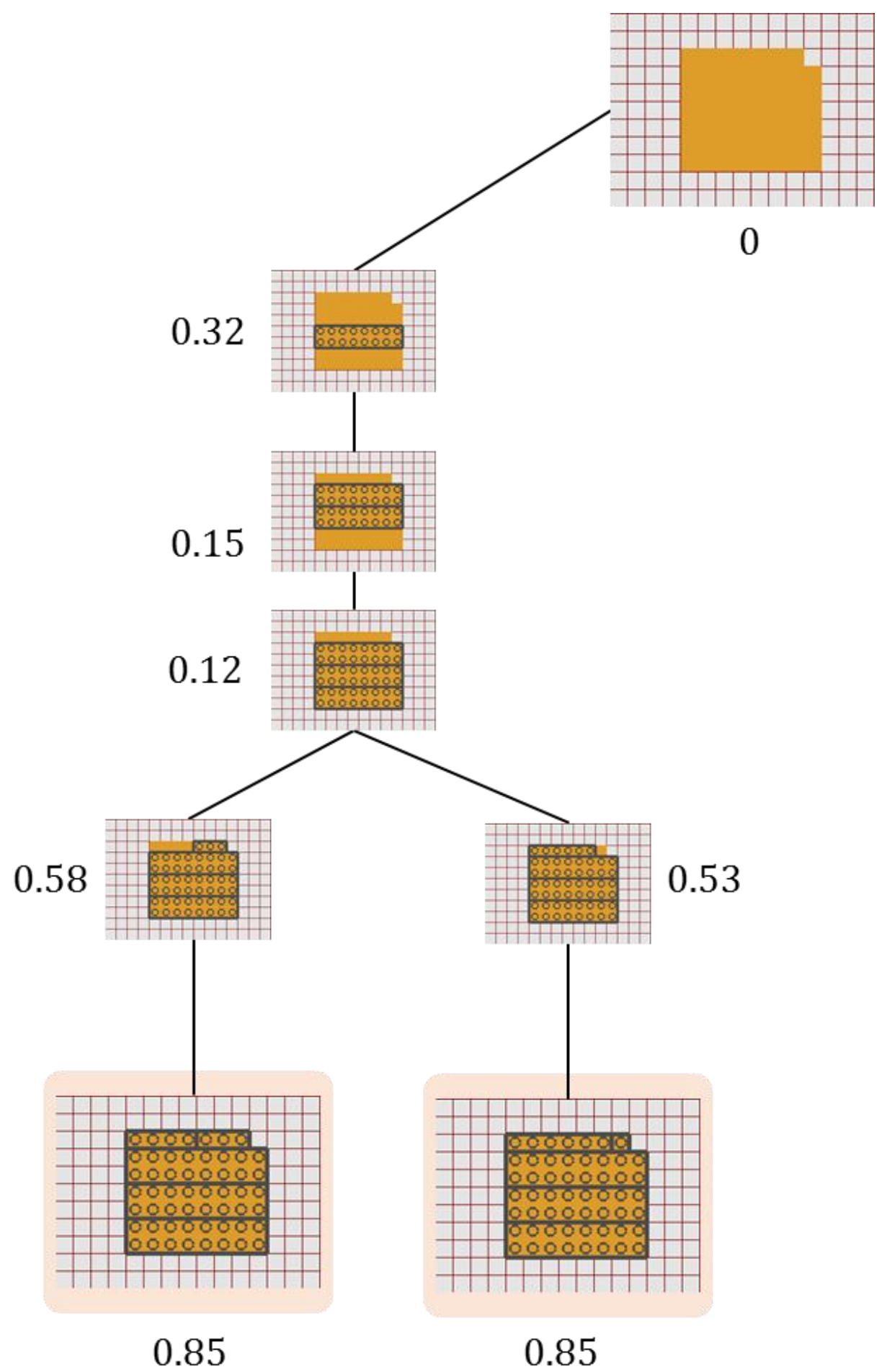

Fig. 12. The non-unique leaf nodes of $A^{*}$ search tree with same minimum values. 


\subsubsection{Comparison with different objectives}

We pursue four different objectives and compare their resulting layouts: (i) balancing three objectives, (ii) stability-oriented, (iii) aesthetics-oriented, and (iv) efficiency-oriented. The weights of the tiling equation in Eqn. (8) are suggested in Fig. 13. The resulting objective values in Eqn. (8) and the number of bricks used in the layout are also suggested in the same figure.

\begin{tabular}{|c|c|c|c|c|c|c|c|c|c|}
\hline & \multicolumn{3}{|c|}{ Objectives } & \multirow{2}{*}{\multicolumn{4}{|c|}{ Weights }} & \multirow{3}{*}{$\begin{array}{l}\text { Total } \\
\left(G\left(L_{k}\right)\right)\end{array}$} & \multirow{3}{*}{$\begin{array}{l}\text { No. of } \\
\text { bricks }\end{array}$} \\
\hline & \multirow{2}{*}{$\frac{\text { Stability }}{g_{s}}$} & \multirow{2}{*}{$\frac{\text { Aesthetic }}{g_{a}}$} & \multirow{2}{*}{$\frac{\text { Efficiency }}{g_{h}}$} & & & & & & \\
\hline & & & & $w_{s}$ & $w_{a}$ & $w_{h}$ & $w_{v}$ & & \\
\hline Balanced & 0.68 & 0.75 & 0.23 & 1 & 1 & 1 & 0 & 1.67 & 13 \\
\hline Stability & 0.613 & 0.8 & 0.31 & 10 & 1 & 1 & 0 & 7.25 & 15 \\
\hline Aesthetics & 0.74 & 0.0 & 0.28 & 1 & 10 & 1 & 0 & 1.03 & 14 \\
\hline Efficiency & 0.76 & 0.27 & 0.22 & 1 & 1 & 10 & 0 & 3.25 & 10 \\
\hline
\end{tabular}

Fig. 13. The parameters for the tiling equation and the result values.

As suggested in Fig. 13, the $g$ term of highest $w$ term has lowest value among the other $w$ terms. $g_{s}$, for example, is lowest in the second row among the four different cases, since $w_{s}$ is highest in the second row. This proves that our minimization process of the tiling equation is valid.

Pursuing different objectives results in different layouts. We present the different layouts in Fig. 14. The processes of developing the layouts are compared in Fig. 15. The leaf nodes of the tree in Fig. 15 correspond to the layouts in Fig. 14.

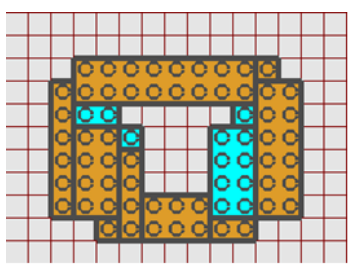

Balanced

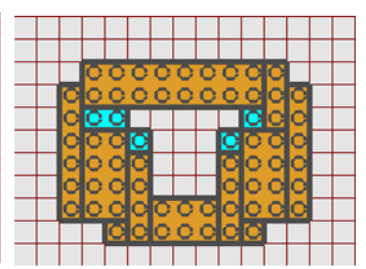

Stability-oriented
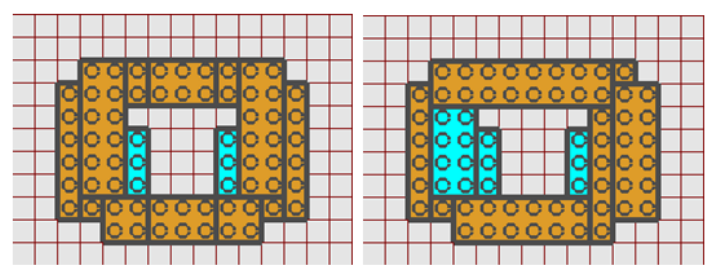

Aesthetic-oriented Efficiency-oriented

Fig. 14. Different layouts are produced from different weights on the objectives in Eqn. (8). 


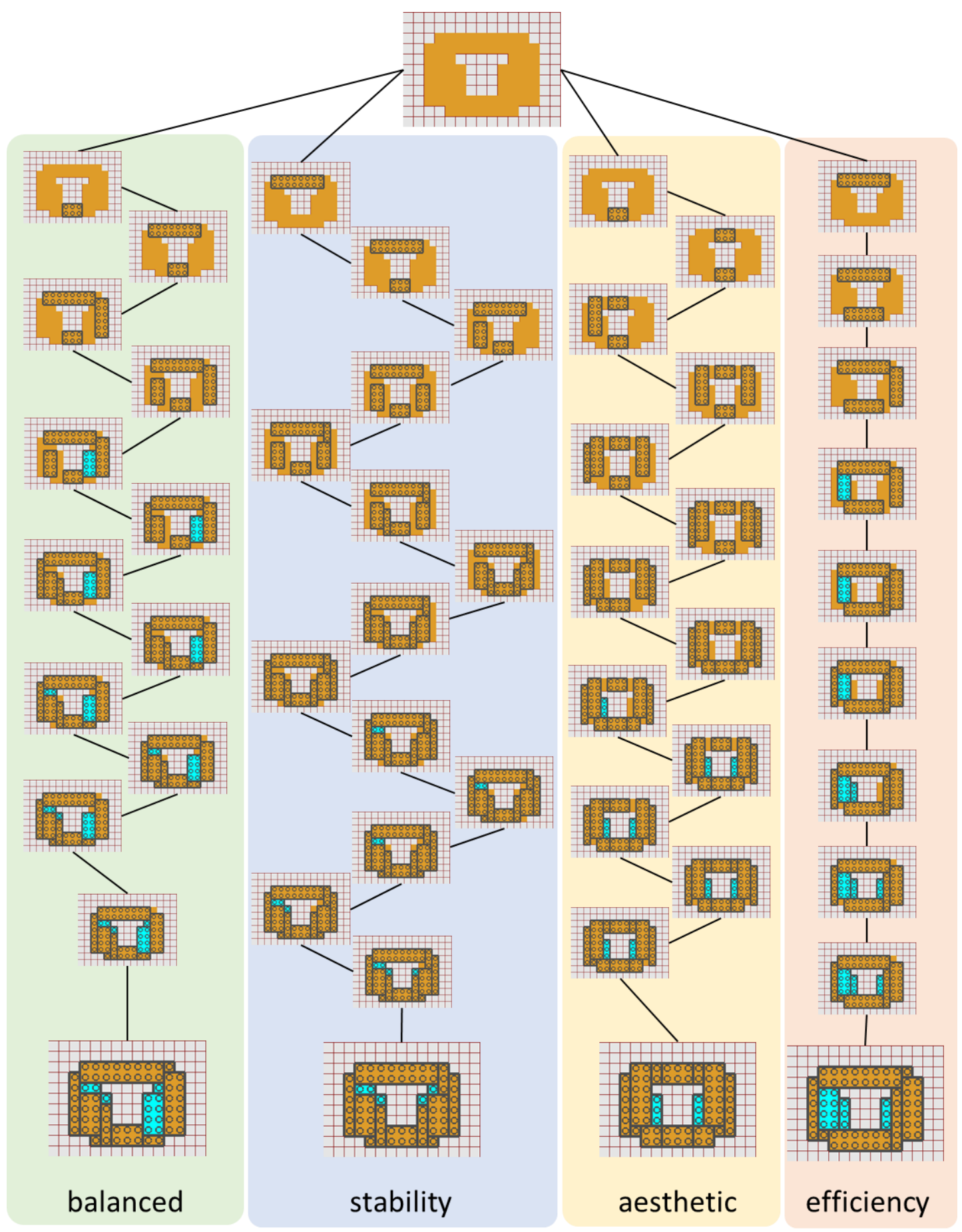

Fig. 15. Comparison of tiling processes according to different weights in Eqn. (8). The layouts in the leaf nodes correspond to those in Fig. 17. 


\section{Implementation and results}

We have implemented our legorization algorithm on a PC with Pentium i7 CPU and 16 GByte main memory. We test our algorithm on five models and produce their assembly maps. The information about our legorization is suggested in Fig. 16. The input models are illustrated in Fig. 17 and their results are illustrated in Fig. 18.

In building the LEGO models, we sometimes needed a supporter bricks for the empty inside area of the castle model.

\begin{tabular}{|l|l|l|l|l|l|}
\hline & \multicolumn{1}{|c|}{$\begin{array}{c}\text { No. of } \\
\text { vertices }\end{array}$} & Resolution & $\begin{array}{c}\text { Time for } \\
\text { legorization }\end{array}$ & $\begin{array}{c}\text { No. of } \\
\text { bricks }\end{array}$ & $\begin{array}{c}\text { Time for } \\
\text { assemble }\end{array}$ \\
\hline Ryan & 26033 & 20 & $16.64 \mathrm{sec}$ & 375 & $3 \mathrm{~h}$ \\
\hline Apeach & 4523 & 20 & $20.6 \mathrm{sec}$ & 343 & $3 \mathrm{~h}$ \\
\hline Leonard & 9720 & 20 & $10.13 \mathrm{sec}$ & 291 & $3 \mathrm{~h}$ \\
\hline Eevee & 2539 & 30 & $43.83 \mathrm{sec}$ & 1402 & $6 \mathrm{~h}$ \\
\hline Castle & 5762 & 20 & $34.25 \mathrm{sec}$ & 715 & $5 \mathrm{~h}$ \\
\hline
\end{tabular}

Fig. 16. Results on legorization.

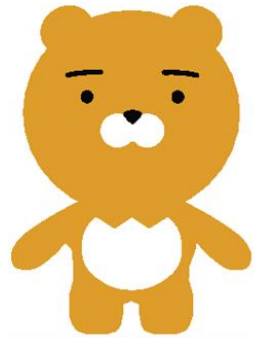

Ryan

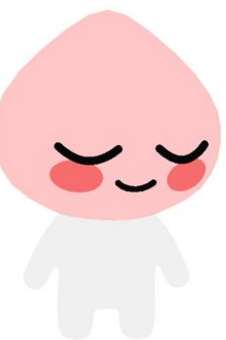

Apeach

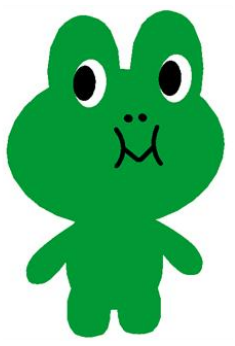

Leonard

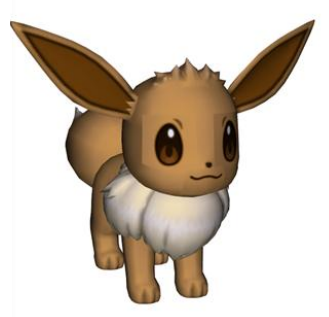

Eevee

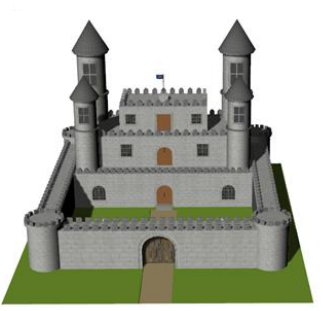

Castle

Fig. 17. Input models of our legorization. 

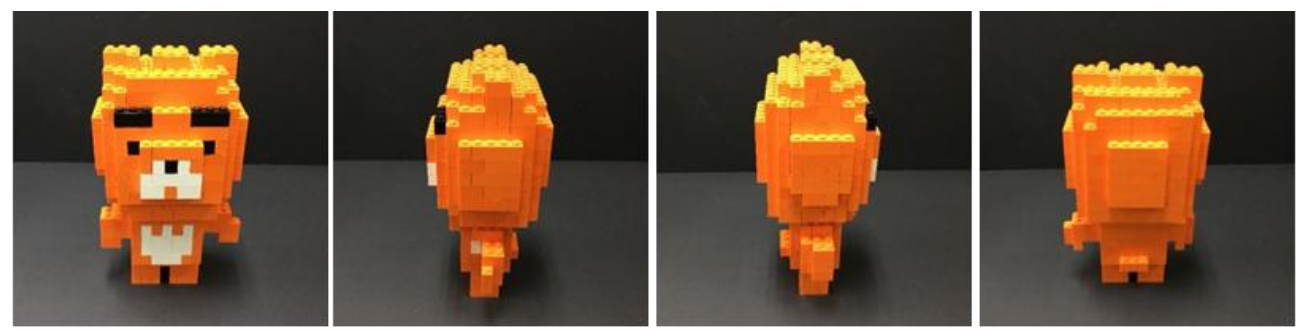

Ryan
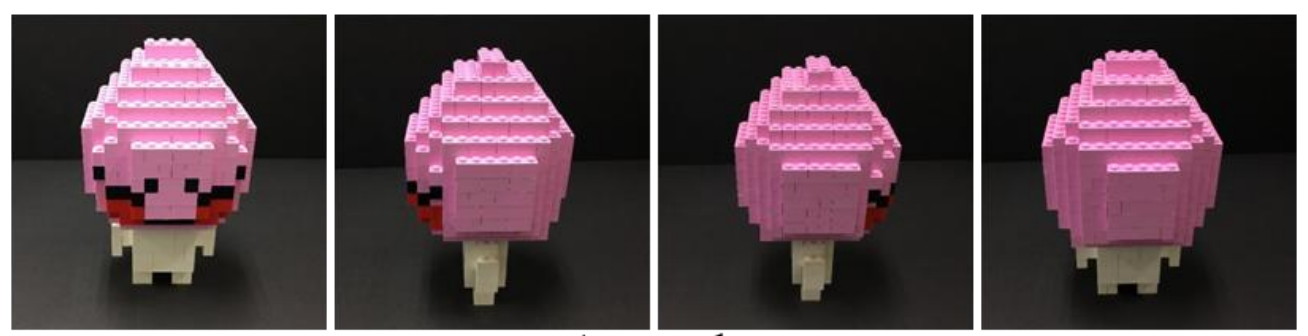

Apeach
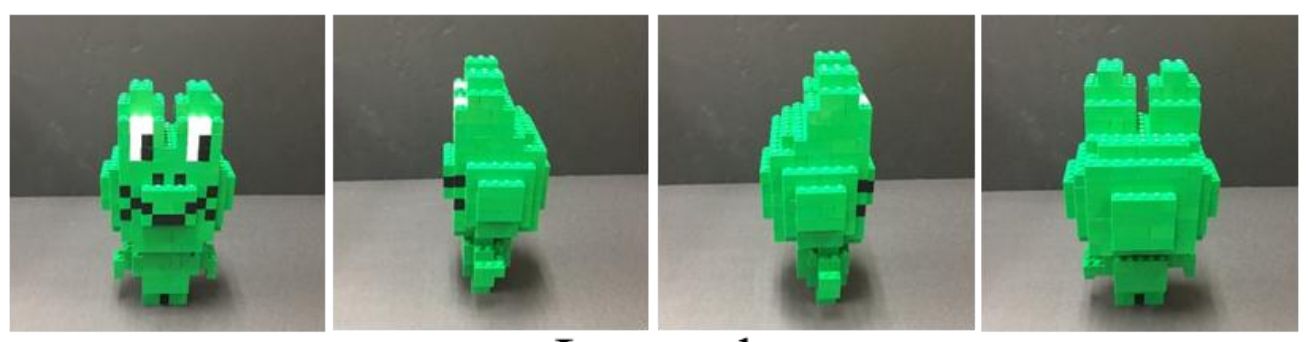

\section{Leonard}
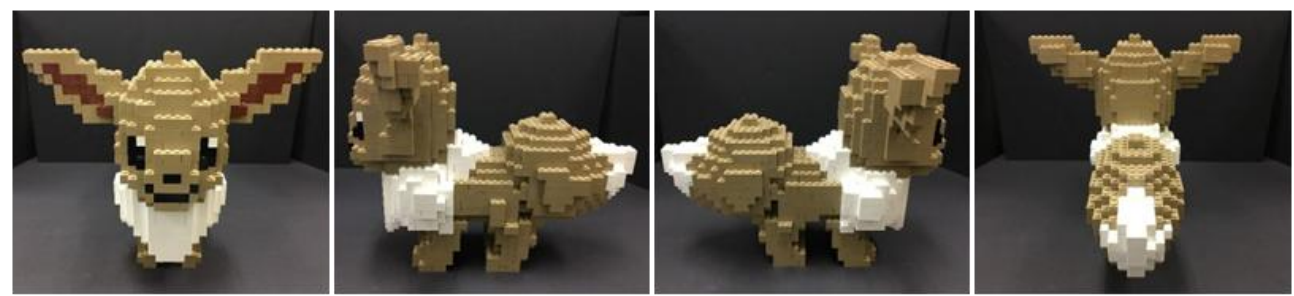

\section{Eevee}
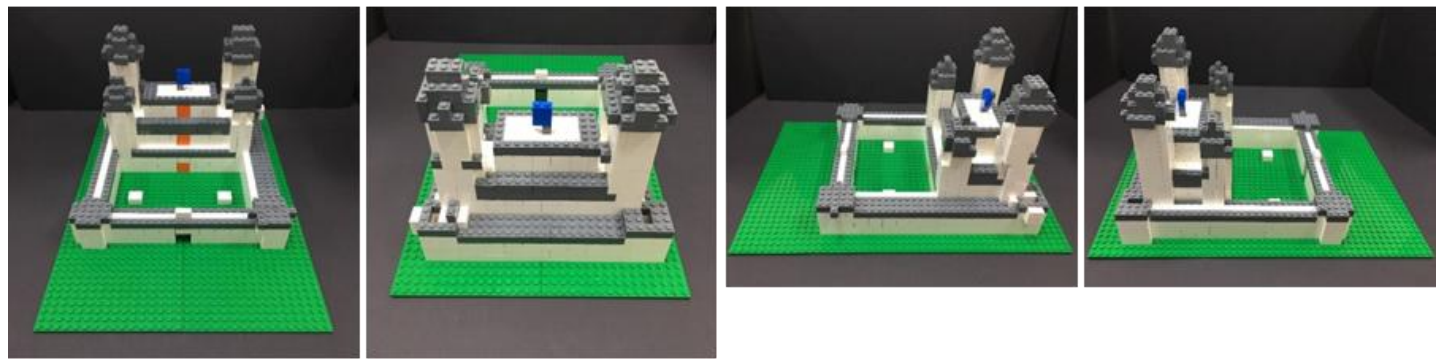

\section{Castle}

Fig. 18. Outputs of our legorization. 


\section{Comparison and discussion}

We compare our legorization objectives with the legorization heuristics presented by Gower et al. [13] and Petrovic [14]. For the comparison, we quantify the stability, aesthetics and efficiency of the layouts from ours and other two heuristics for the 99 layers of five models. We summarize the results of comparison in Fig. 19 and illustrate the results in Fig. 20. According to the comparison, our approach shows better stability, aesthetics and efficiency than the widely-used two legorization heuristics.

\begin{tabular}{|c|c|c|c|c|c|}
\hline Model & Layer & Better & Tie & Worse & Better\% \\
\hline Ryan & 20 & 18 & 1 & 1 & 94.4 \\
\hline Apeach & 20 & 18 & 1 & 1 & 88.9 \\
\hline Leonard & 20 & 17 & 0 & 3 & 89.5 \\
\hline Eevee & 30 & 23 & 2 & 5 & 88.9 \\
\hline Castle & 19 & 15 & 2 & 2 & 88.2 \\
\hline
\end{tabular}

Fig. 19. Summary of the comparison for each model. Our approach shows better results for $88.2 \%$ $94.4 \%$.

\section{Conclusion and future work}

We have presented a legorization framework that converts a 3D mesh into a LEGO model by producing its assembly diagram. Our framework preserves the shape of input model by proposing a silhouette-fitting scheme and presents a legorization scheme satisfying stability, aesthetics and efficiency. Our framework allows multi-height bricks which improve the efficiency of the model. We have tested our scheme for five models including characters and buildings.

As a future work, we plan to build a legorization framework that uses special bricks as well as various multi-height bricks. Furthermore, we are going to include estimating loads on a brick using our two-colored graph model.

\section{Acknowledgement}

This research was supported by a research grant from Sangmyung Univ. in 2015. 


\begin{tabular}{|c|r|r|r|r|}
\hline \multicolumn{5}{|c|}{ Ryan } \\
\hline \multirow{2}{*}{ Layer } & \multicolumn{3}{|c|}{ Heuritics } \\
\cline { 2 - 4 } & Ours & {$[13]$} & {$[14]$} & \\
\hline 1 & 0.77 & 1.77 & 1.77 & 1.00 \\
\hline 2 & 0.77 & 0.77 & 0.77 & 0.00 \\
\hline 3 & 0.72 & 1.60 & 1.60 & 0.88 \\
\hline 4 & 1.54 & 1.54 & 1.54 & 0.00 \\
\hline 5 & 2.09 & 1.91 & 1.91 & -0.18 \\
\hline 6 & 1.25 & 2.05 & 1.83 & 0.69 \\
\hline 7 & 1.12 & 1.06 & 1.36 & 0.09 \\
\hline 8 & 1.09 & 1.87 & 1.87 & 0.78 \\
\hline 9 & 0.85 & 1.24 & 1.21 & 0.38 \\
\hline 10 & 0.29 & 1.39 & 1.42 & 1.12 \\
\hline 11 & 1.14 & 2.01 & 1.62 & 0.68 \\
\hline 12 & 1.14 & 1.32 & 1.48 & 0.26 \\
\hline 13 & 1.07 & 1.52 & 1.60 & 0.49 \\
\hline 14 & 1.17 & 1.73 & 1.76 & 0.58 \\
\hline 15 & 1.02 & 1.38 & 1.44 & 0.39 \\
\hline 16 & 1.08 & 1.21 & 1.23 & 0.16 \\
\hline 17 & 1.08 & 1.41 & 1.41 & 0.33 \\
\hline 18 & 1.96 & 2.02 & 1.99 & 0.05 \\
\hline 19 & 1.45 & 1.59 & 1.62 & 0.19 \\
\hline 20 & 1.22 & 1.56 & 1.59 & 0.36 \\
\hline & & & & \\
\hline
\end{tabular}

\begin{tabular}{|l|l|l|l|l|}
\hline \multicolumn{4}{|c|}{ Leonard } \\
\hline \multirow{2}{*}{ Layer } & \multicolumn{3}{|c|}{ Heuritics } & \multirow{2}{*}{ Diff. } \\
\cline { 2 - 4 } & Ours & {$[13]$} & {$[14]$} & \\
\hline
\end{tabular}

\begin{tabular}{|l|l|l|l|l|l|l|l|l|l|}
\hline 1 & 0.80 & 0.80 & 0.80 & 0.00 & 25 & 1.76 & 2.16 & 2.16 & 0.40 \\
\hline
\end{tabular}

\begin{tabular}{|l|l|l|l|l|l|l|l|l|l|}
\hline 2 & 0.82 & 1.73 & 1.73 & 0.91 & 26 & 1.90 & 2.15 & 2.22 & 0.29 \\
\hline
\end{tabular}

\begin{tabular}{|l|l|l|l|l|l|l|l|l|l|}
\cline { 4 - 9 }$n$ & 1.13 & 0.62 & 0.62 & -0.51 & 27 & 2.30 & 2.11 & 2.24 & -0.13 \\
\hline
\end{tabular}

\begin{tabular}{|r|r|r|r|r|r|r|r|r|r|}
\hline 4 & 1.39 & 1.21 & 1.21 & -0.18 & 28 & 2.21 & 2.30 & 2.30 & 0.09 \\
\hline 5 & 1.75 & 1.77 & 1.77 & 0.02 & 29 & 1.17 & 2.22 & 2.22 & 1.05 \\
\hline
\end{tabular}

\begin{tabular}{|l|l|l|l|l|l|l|l|l|l|}
\hline 6 & 1.95 & 2.07 & 2.07 & 0.12 & 30 & 1.47 & 1.47 & 1.47 & 0.00 \\
\hline
\end{tabular}

\begin{tabular}{|l|l|l|l|l|}
\hline 7 & 1.19 & 2.17 & 2.17 & 0.98 \\
\hline
\end{tabular}

\begin{tabular}{|l|l|l|l|l|}
\hline 8 & 0.77 & 0.94 & 0.94 & 0.17 \\
\hline 9 & 0.27 & 1.43 & 1.43 & 1.16 \\
\hline
\end{tabular}

\begin{tabular}{|c|r|r|r|r|}
\hline 9 & 0.27 & 1.43 & 1.43 & 1.16 \\
\hline 10 & 1.06 & 1.59 & 1.59 & 0.53 \\
\hline
\end{tabular}

\begin{tabular}{|l|l|l|l|l|}
\hline 11 & 1.04 & 1.20 & 1.20 & 0.16 \\
\hline
\end{tabular}

\begin{tabular}{|l|l|l|l|l|}
\hline 12 & 1.17 & 1.76 & 1.52 & 0.47 \\
\hline
\end{tabular}

\begin{tabular}{|l|l|l|l|l|}
\hline 13 & 1.07 & 1.22 & 1.17 & 0.13 \\
\hline 14 & 0.99 & 1.17 & 1.17 & 0.18 \\
\hline
\end{tabular}

\begin{tabular}{|l|r|r|r|r|}
\hline 14 & 0.99 & 1.17 & 1.17 & 0.18 \\
\hline 15 & 1.58 & 1.72 & 1.72 & 0.14 \\
\hline
\end{tabular}

\begin{tabular}{|l|l|l|l|l|}
\hline 16 & 1.63 & 1.70 & 1.61 & 0.03 \\
\hline
\end{tabular}

\begin{tabular}{|l|r|r|r|r|}
\hline 17 & 0.98 & 1.63 & 1.64 & 0.66 \\
\hline 18 & 1.80 & 1.58 & 1.58 & -0.22 \\
\hline
\end{tabular}

\begin{tabular}{|l|l|l|l|l|}
\hline 18 & 1.80 & 1.58 & 1.58 & -0.22 \\
\hline
\end{tabular}

\begin{tabular}{l|l|l|l|l|}
\hline 19 & 0.78 & 0.87 & 0.87 & 0.09 \\
\hline 20 & 0.92 & 0.92 & 0.92 & 0.00 \\
\hline
\end{tabular}

\begin{tabular}{|c|c|c|c|c|}
\hline \multicolumn{5}{|c|}{ Apeach } \\
\hline \multirow{2}{*}{ Layer } & \multicolumn{3}{|c|}{ Heuritics } & \multirow{2}{*}{ Diff. } \\
\hline & Ours & [13] & [14] & \\
\hline 1 & 0.80 & 0.80 & 0.80 & 0.00 \\
\hline 2 & 0.80 & 0.80 & 0.80 & 0.00 \\
\hline 3 & 0.27 & 0.27 & 0.27 & 0.00 \\
\hline 4 & 0.69 & 0.69 & 0.69 & 0.00 \\
\hline 5 & 0.98 & 0.98 & 0.98 & 0.00 \\
\hline 6 & 1.09 & 1.09 & 1.09 & 0.00 \\
\hline 7 & 1.19 & 1.19 & 1.19 & 0.00 \\
\hline 8 & 0.80 & 0.63 & 0.63 & -0.17 \\
\hline 9 & 0.50 & 0.55 & 0.55 & 0.05 \\
\hline 10 & 1.21 & 1.91 & 2.00 & 0.75 \\
\hline 11 & 1.21 & 1.61 & 1.31 & 0.25 \\
\hline 12 & 1.39 & 1.50 & 1.49 & 0.11 \\
\hline 13 & 1.30 & 1.31 & 1.34 & 0.03 \\
\hline 14 & 1.18 & 1.28 & 1.28 & 0.10 \\
\hline 15 & 1.22 & 1.53 & 1.26 & 0.18 \\
\hline 16 & 0.95 & 1.12 & 1.08 & 0.15 \\
\hline 17 & 0.96 & 0.94 & 0.94 & -0.02 \\
\hline 18 & 0.83 & 0.83 & 0.83 & 0.00 \\
\hline 19 & 0.39 & 0.39 & 0.39 & 0.00 \\
\hline 20 & 1.14 & 1.14 & 1.14 & 0.00 \\
\hline \multicolumn{5}{|c|}{ Castle } \\
\hline \multirow{2}{*}{ Layer } & \multicolumn{3}{|c|}{ Heuritics } & \multirow{2}{*}{ Diff. } \\
\hline & Ours & [13] & [14] & \\
\hline 1 & 1.47 & 1.59 & 1.59 & 0.12 \\
\hline 2 & 1.68 & 1.75 & 1.78 & 0.09 \\
\hline 3 & 1.69 & 1.79 & 1.76 & 0.09 \\
\hline 4 & 1.43 & 1.82 & 1.80 & 0.38 \\
\hline 5 & 2.36 & 2.39 & 2.38 & 0.03 \\
\hline 6 & 2.00 & 2.20 & 2.28 & 0.24 \\
\hline 7 & 1.26 & 1.66 & 1.68 & 0.41 \\
\hline 8 & 1.62 & 2.10 & 1.91 & 0.39 \\
\hline 9 & 2.16 & 2.22 & 2.22 & 0.06 \\
\hline 10 & 1.73 & 2.03 & 1.99 & 0.28 \\
\hline 11 & 1.53 & 1.75 & 1.73 & 0.21 \\
\hline 12 & 2.53 & 2.43 & 2.40 & -0.12 \\
\hline 13 & 1.95 & 1.87 & 1.93 & -0.05 \\
\hline 14 & 2.02 & 2.11 & 2.14 & 0.11 \\
\hline 15 & 1.72 & 2.05 & 2.05 & 0.33 \\
\hline 16 & 1.27 & 1.39 & 1.39 & 0.12 \\
\hline 17 & 2.22 & 2.12 & 2.12 & -0.10 \\
\hline 18 & 2.04 & 1.60 & 1.60 & -0.44 \\
\hline 19 & 2.11 & 2.11 & 2.11 & 0.00 \\
\hline
\end{tabular}

Fig. 20. Comparison of our heuristic with Gower's [13] and Petrovic's [14]. The white row shows our approach is better and the red row shows our approach is worse. The pink row shows they are tied. 


\section{Reference}

[1] A. Kaufman, "Efficient algorithms for 3d scan-conversion of parametric curves, surfaces, and volumes," ACM Computer Graphics, 171-179, 21 (4), 1987. Article (CrossRef Link)

[2] H. A. Kaufman, and E. Shimony, "3d scan-conversion algorithms for voxel-based graphics," in Proc. of Interactive 3D graphics 1986, pp. 45-75, 1986. Article (CrossRef Link)

[3] H. Chen, and S. Fang, "Fast voxelization of three-dimensional synthetic objects," Journal of Graphics Tools, pp. 33-45, 3 (4), 1998. Article (CrossRef Link)

[4] S. Fang, and H. Chen, "Hardware accelerated voxelization," Computers \& Graphics, pp. 433-442, 24 (3), 2000. Article (CrossRef Link)

[5] E.-A. Karabassi, G. Papaioannou, and T. Theoharis, "A fast depth-buffer-based voxelization algorithm," Journal of Graphics Tools, pp. 5-10, 4 (4), 1999. Article (CrossRef Link)

[6] G. Passalis, I. A. Kakadiaris, and T. Theoharis, "Efficient hardware voxelization," in Proc. of CGI 2004, pp. 374-377, 2004,. Article (CrossRef Link)

[7] E. Eisemann, and X. Decoret, "Fast scene voxelization and applications," in Proc. of Interactive 3 D graphics and games 2006, pp. 71-78, 2006. Article (CrossRef Link)

[8] E. Eisemann, and X. Decoret, "Single-pass GPU solid voxelization for real-time applications," in Proc. of Graphics Interface 2008, pp. 73-80, 2008. Article (CrossRef Link)

[9] L. Silva, V. Pamplona, and J. Comba, "Legolizer: A realtime system for modeling and rendering lego representations of boundary models," in Proc. of Brazilian Symposium on Computer Graphics and Image Processing 2009, pp. 17-23, 2009. Article (CrossRef Link)

[10] F. S. Nooruddin, and G. Turk, "Simplification and repair of polygonal models using volumetric techniques," IEEE Transactions on Visualization and Computer Graphics, pp. 191-205, 9(2), 2003. Article (CrossRef Link)

[11] F. Q. W. Hong, and A. Kaufman, "Gpu-based object-order ray-casting for large datasets," in Proc. of 4th International Workshop on Volume Graphics 2005, pp. 177-185, 2005. Article (CrossRef Link)

[12] M. Schwarz, H. P. Seidel, "Fast parallel surface and solid voxelization on GPUs," $A C M$ Transactions on Graphics, pp. 179, 29 (6), 2010. Article (CrossRef Link)

[13] R. Gower, A. Heydtmann, and H. Petersen, "Lego: Automated model construction," in Proc. of 32nd European Study Group with Industry 1998, pp. 81-94, 1998. Article (CrossRef Link)

[14] P. Petrovic, "Solving lego brick layout problem using evolutionary algorithms," in Proc. of Norwegian Conference on Computer Science, pp. 87-97, 2001.

[15] L. van Zijl, and E. Smal, "Cellular automata with cell clustering," in Proc. Of Automata 2008, pp. 425-440, 2008.

[16] E. Smal, "Automated brick sculpture construction, Ph.D. thesis," Stellenbosch: Stellenbosch University, 2008. Article (CrossRef Link)

[17] R. Testuz, Y. Schwartzburg, and M. Pauly, "Automatic generation of constructable brick sculptures," in Proc. of Eurographics (Short Papers) 2013, pp. 81-84, 2013. Article (CrossRef Link)

[18] S. Ono, A. Andre, and Y. Chang, "Automatic generation of lego from the polygonal data," in Proc. of International workshop on advanced image technology 2013, pp. 262-267, 2013. Article (CrossRef Link)

[19] M. Zhang, J. Mitani, Y. Kanamori, and Y. Fukui, "Blocklizer: Interactive design of stable mini block artwork," in Proc. of ACM SIGGRAPH 2014 Posters, p. 18, 2014. Article (CrossRef Link)

[20] J. W. Kim, K. K. Kang, and J. H. Lee, "Survey on automated lego assembly construction," in Proc. of WSCG 2014, pp. 89-96, 2014.

[21] S. Lee, J. Kim, J. W. Kim, B. and R. Moon, "Finding an optimal lego® brick layout of voxelized 3d object using a genetic algorithm," in Proc. of the 2015 Annual Conference on Genetic and Evolutionary Computation, pp. 1215-1222, 2015. Article (CrossRef Link) 
[22] M. Zhang, Y. Igarashi, Y. Kanamori, and J. Mitani, "Designing mini block artwork from colored mesh," in Proc. of Smart Graphics 2015, p. 2, 2015. Article (CrossRef Link)

[23] B. Stephenson, "A multi-phase search approach to the lego construction problem," in Proc. of Ninth Annual Symposium on Combinatorial Search 2016, pp. 89-97, 2016.

[24] M. Zhang, Y. Igarashi, Y. Kanamori, and J. Mitani, "Component-based building instructions for block assembly," in Proc. of Computer-Aided Design and Applications 2016, pp. 55-59, 2016. Article (CrossRef Link)

[25] J. Hong, D. Way, Z. Shih, W. Tai, and C. C. Chang, "Inner engraving for the creation of a balanced lego sculpture,” The Visual Computer, pp. 569-578, 32 (5), 2016. Article (CrossRef Link)

[26] S. J. Luo, Y. Yue, C. K. Huang, Y. H. Chung, S. Imai, T. Nishita, and B. Y. Chen, "Legolization: Optimizing lego designs," ACM Transactions on Graphics, pp. 222, 34 (6), 2015. Article (CrossRef Link)

[27] T. Kozaki, H. Tedenuma, and T. Maekawa, "Automatic generation of lego building instructions from multiple photographic images of real objects," Computer-Aided Design, pp. 13-22, 70 (C), 2016. Article (CrossRef Link)

[28] H. Freeman, "Computer processing of line drawing images," ACM Computing Surveys, pp. 57-97, 6 (1), 1974. Article (CrossRef Link)

[29] G. Rong and T. Tan, "Jump flooding in gpu with applications to voronoi diagram and distance transform," in Proc. of ACM Symposium on Interactive 3D graphics and Games, pp. 109-116, 2006. Article (CrossRef Link)

\section{Appendix}

The list of heuristics proposed by Gower et al. [13] is as follows:

1. Cover ratio: The ratio of the cells covered by the bricks in the incident layer to all cells in a layer.

2. Big brick: The bigger brick is favored to the smaller bricks. It can be estimated as the number of bricks per the area covered by the bricks.

3. Perpendicularity: The number of bricks perpendicular to the bricks in the incident layer.

4. Vertical boundary: The edge covered by the bricks in the incident layer.

5. T-shaped joining: The distance between an edge and the center of its adjacent brick in the same layer.

6. Covered edge by center: The distance between an edge and the center of its covering brick in the incident layer.

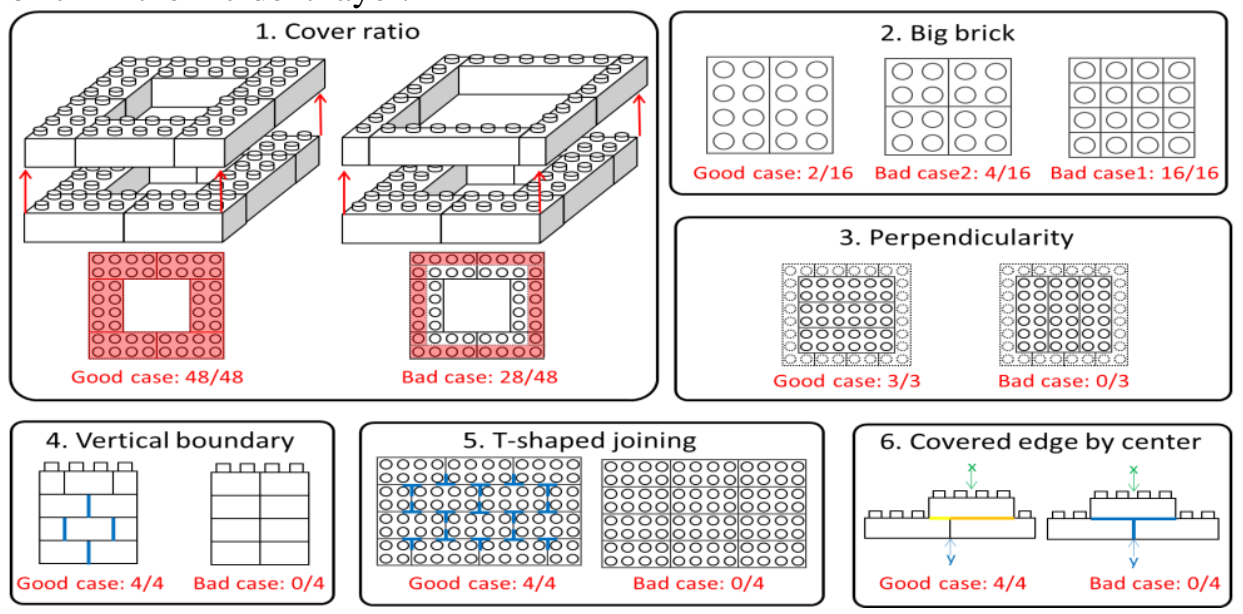

Fig. 21. Six classical heuristic proposed by Gower et al. [13]. 

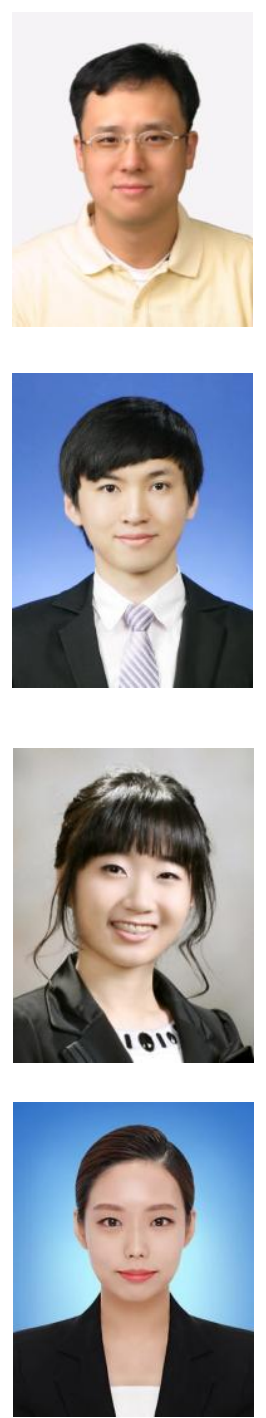

Kyungha Min received his MS in Computer Science from KAIST in 1992. He received his BS and Ph.D in Computer Science and Engineering from POSTECH in 1994 and 2000, respectively. His main research interests are computer graphics and image processing.

Cheolseong Park received his B.S. degree from Sangmyung University, Seoul, Korea, in 2016. He is currently a graduate student in the same college. His research interests are computer graphics. He is also interested in image processing, 3D-mesh processing and deep learning.

Heekyung Yang received her B.S. degree and M.S. degree from Sangmyung University, Seoul, Korea, in 2010 and 2012, respectively. She is currently a Ph.D. student in the same college. Her research interests are computer graphics, especially NPR (non-photorealistic rendering). She is also interested in image processing, 3D-mesh processing, and deep learning.

Grim Yun received her B.S. degree and M.S. degree from Sangmyung University, Seoul, Korea, in 2017. Her research interests are computer graphics. She is also interested in image processing, 3D-mesh processing and deep learning. 